\title{
Bulk Terpolymerization of Conjugated Linoleic Acid with Styrene and Butyl Acrylate
}

Stéphane Roberge, Marc A. Dubé*

Department of Chemical and Biological Engineering, Centre for Catalysis Research and Innovation, University of Ottawa, 161 Louis Pasteur Pvt., Ottawa, ON, K1N 6N5 Canada

* Marc.Dube@uOttawa.ca

\section{Supporting Information: Matlab Model}

4 pages, 1 table 
Matlab version: Mathworks, R2014b, 8.4.0.150421, 64-bit (maci64)

The following function "f3" was created:

function $\mathrm{rk}=\mathrm{f} 3(\mathrm{t}, \mathrm{y})$

$\mathrm{kd}=2.47 \mathrm{E}-5 ; \% \mathrm{~s}-1$ for $\mathrm{BPO}$ at $80 \mathrm{deg} \mathrm{C}$

Izero $=0.07 ; \% \mathrm{~mol} / \mathrm{L}$ initiator $(2 \mathrm{phm} \mathrm{w} / \mathrm{w})$

Idot=Izero*exp $(-\mathrm{kd} * \mathrm{t}) ; \% \mathrm{~mol} / \mathrm{L}$ initiator radicals

$\mathrm{kiA}=0.0003 ; \% \mathrm{~L}$ mol-1 sec-1

$\mathrm{kiB}=0$;

$\mathrm{kiC}=0$;

$\mathrm{kiO}=0$;

$\mathrm{kpAA}=0.03$

$\mathrm{kpAB}=0.3$;

$\mathrm{kpAC}=0.6$;

$\mathrm{kpBA}=0.03$;

$\mathrm{kpBB}=341$;

$\mathrm{kpBC}=357$;

$\mathrm{kpCA}=0.3$;

$\mathrm{kpCB}=15205$;

$\mathrm{kpCC}=3024$;

$\mathrm{ktrAO}=1000$

$\mathrm{ktrBO}=1000$

$\mathrm{ktrCO}=2000$;

$\mathrm{a}=-\mathrm{kiA}^{*} \operatorname{Idot}^{*} \mathrm{y}(1)-\mathrm{kpAA}{ }^{*} \mathrm{y}(5) * \mathrm{y}(1)-\mathrm{kpBA}^{*} \mathrm{y}(6) * \mathrm{y}(1)-\mathrm{kpCA}^{*} \mathrm{y}(7) * \mathrm{y}(1) ; \%$ change of CLA 
$b=-k_{B} * \operatorname{Idot}^{*} y(2)-\mathrm{kpAB}^{*} \mathrm{y}(5) * \mathrm{y}(2)-\mathrm{kpBB}^{*} \mathrm{y}(6) * \mathrm{y}(2)-\mathrm{kpCB}^{*} \mathrm{y}(7) * \mathrm{y}(2) ; \%$ change of Sty

$\mathrm{c}=-\mathrm{kiC}^{*} \operatorname{Idot}^{*} \mathrm{y}(3)-\mathrm{kpAC} \mathrm{C}^{*}(5) * \mathrm{y}(3)-\mathrm{kpBC} * \mathrm{y}(6) * \mathrm{y}(3)-\mathrm{kpCC} * \mathrm{y}(7) * \mathrm{y}(3) ; \%$ change of BA

$\mathrm{d}=-\mathrm{kiO}^{*} \operatorname{Idot}^{*} \mathrm{y}(4)-\mathrm{ktrAO} * \mathrm{y}(5) * \mathrm{y}(4)-\mathrm{ktrBO} * \mathrm{y}(6) * \mathrm{y}(4)-\mathrm{ktrCO} * \mathrm{y}(7) * \mathrm{y}(4) ; \%$ change of activated

Oleic

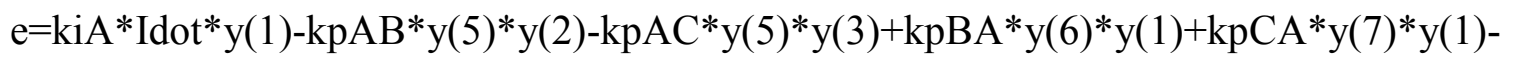

$\mathrm{ktrAO}^{*} \mathrm{y}(5)^{*} \mathrm{y}(4) ; \%$ ending in CLA

$f=k i B^{*} \operatorname{Idot} * y(2)+k p A B * y(5) * y(2)-k p B A * y(6) * y(1)-k p B C * y(6) * y(3)+k p C B * y(7) * y(2)-$

ktrBO*y(6)*y(4); \%ending in Sty

$\mathrm{g}=\mathrm{kiC}^{*} \operatorname{Idot}^{*} \mathrm{y}(3)+\mathrm{kpAC} * \mathrm{y}(5) * \mathrm{y}(3)+\mathrm{kpBC} * \mathrm{y}(6) * \mathrm{y}(3)-\mathrm{kpCA}^{*} \mathrm{y}(7) * \mathrm{y}(1)-\mathrm{kpCB}^{*} \mathrm{y}(7)^{*} \mathrm{y}(2)-$

$\mathrm{ktrCO} * \mathrm{y}(7) * \mathrm{y}(4) ; \%$ ending in $\mathrm{BA}$

$\mathrm{rk}=[\mathrm{a} ; \mathrm{b} ; \mathrm{c} ; \mathrm{d} ; \mathrm{e} ; \mathrm{f} ; \mathrm{g}]$;

The following "m file" was created as the main program:

time $=0: 1: 70000$

initial $=[1.6360,0.4469,1.7794,0.6338,0,0,0]$;

[t,y]=ode45(@f3, time, initial);

figure;

$\operatorname{plot}\left(t, y(:, 1), y^{\prime}\right)$;

title('Models'), xlabel('seconds'), ylabel('mol/L');

hold on;

$\operatorname{plot}\left(t, y(:, 2), \mathrm{m}^{\prime}\right)$;

$\operatorname{plot}\left(t, y(:, 3),{ }^{\prime}{ }^{\prime}\right)$;

$\operatorname{plot}\left(\mathrm{t}, \mathrm{y}(:, 4), \mathrm{r}^{\prime}\right)$; 
$\operatorname{plot}\left(\mathrm{t}, \mathrm{y}(:, 5), \mathrm{g}^{\prime}\right)$;

$\operatorname{plot}\left(\mathrm{t}, \mathrm{y}(:, 6), \mathrm{b}^{\prime}\right)$;

$\operatorname{plot}\left(\mathrm{t}, \mathrm{y}(:, 7), \mathrm{k}^{\prime}\right)$;

legend('CLA','Sty','BA', 'Oleic', 'Ra', 'Rb', 'Rc');

hold off;

The mol/L of CLA, Sty, BA and oleic acid were plotted as a function of time for a quick assessment when possible. Time was varied from 0 to $70,000 \mathrm{~s}, \mathrm{R}_{\mathrm{A}}, \mathrm{R}_{\mathrm{B}}, \mathrm{R}_{\mathrm{C}}$ were initialized for all feeds as 0 and A, B, C, and $\mathrm{O}$ were initialized for each feed as follows (see Table S1):

Table S1. Feed concentrations for Matlab modeling

\begin{tabular}{cccccc}
\hline Run & CLA & Sty & BA & Oleic effective & Initiator \\
& $(\mathrm{mol} / \mathrm{L})$ & $(\mathrm{mol} / \mathrm{L})$ & $(\mathrm{mol} / \mathrm{L})$ & $(\mathrm{mol} / \mathrm{L})$ & $(\mathrm{mol} / \mathrm{L})$ \\
\hline 1 & 0.4352 & 0.5908 & 4.7206 & 0.3151 & 0.07 \\
\hline 3 & 0.8071 & 0.5431 & 3.8348 & 0.3852 & 0.07 \\
4 & 1.1223 & 0.5065 & 3.0494 & 0.4845 & 0.07 \\
5 & 1.4100 & 0.4777 & 2.3999 & 0.4854 & 0.07 \\
6 & 1.6360 & 0.4469 & 1.7794 & 0.6338 & 0.07 \\
7 & 1.8614 & 0.4243 & 1.2655 & 0.6408 & 0.07 \\
8 & 2.1906 & 0.3735 & 0.3733 & 0.8487 & 0.07 \\
\hline
\end{tabular}

